\title{
Hemocompatibility of zwitterionic interfaces and membranes
}

\author{
Mei-Chan Sin, Sheng-Han Chen and Yung Chang \\ This focused review addresses the fundamental development of nonfouling zwitterionic poly(sulfobetaine methacrylate) \\ (polySBMA) coatings on a wide range of interfaces and membranes in our laboratory for use in the development of \\ hemocompatible medical devices. The molecular designs of zwitterionic interfaces (for example, surface packing density, \\ grafting coverage, polymer chain conformation and chemistry) as well as the evolution of 'intelligent' interfaces are scrutinized \\ and discussed. Correlations between polymeric membrane surface modification methods, such as thermal- and plasma-induced \\ polymerizations, the conformation of grafted polySBMA chains and hemocompatibility features, are also delineated. \\ Polymer Journal (2014) 46, 436-443; doi:10.1038/pj.2014.46; published online 18 June 2014
}

Keywords: hemocompatibility; interface; membrane; zwitterionic polysulfobetaine

\section{INTRODUCTION}

Stable blood compatibility of biomaterials used in contact with human whole blood is highly desirable for blood-inert devices. The development of nonfouling surfaces is therefore critically important in biomedical applications that involve human blood-contacting materials. ${ }^{1,2}$ However, only a very limited number of synthetic biomaterials are regarded as good, hemocompatible candidates. It has been suggested that a nanoscale homogenous mixture of balanced charged groups from polyzwitterionic materials is the key to controlling nonfouling properties. An important characteristic of a zwitterionic structure is that it should have both a positively and a negatively charged moiety within the same side chain segment while maintaining overall charge neutrality. A set of functional properties is generally used to guide the design of new nonfouling polymer brushes for contact with human blood: they should be hydrophilic and electrically neutral and hydrogen bond acceptors rather than hydrogen bond donors. ${ }^{3}$ Polyzwitterionic materials can be further classified into polybetaines, such as 2-methacryloyloxylethyl phosphorylcholine (MPC), sulfobetaine methacrylate (SBMA) and carboxybetaine methacrylate. The advantages of nonfouling zwitterionic materials include their simple synthesis, ease of applicability, abundance of raw materials and the availability of functional groups. Our laboratory mainly focuses on the study of zwitterionic polySBMA, emphasizing fundamental molecular designs as well as the development of practical biomedical applications.

\section{DEVELOPMENT OF NONFOULING POLYMERS}

Throughout the development of nonfouling materials, three generations of antifouling polymers have evolved and have been extensively studied: 2-hydroxyethyl methacrylate (HEMA)-based polymers, PEGylated-based polymers and zwitterionic-based polymers. The chemical structures of these polymers are depicted in Figure 1.

\section{FIRST GENERATION: HEMA-BASED SYSTEMS}

The first-generation materials, which derived their fouling resistance from their hydrophilicity, were polymerized from hydroxy-functional methacrylates such as HEMA that are neutral, hydroxyl-rich monomers. Notably, polyHEMA has an abundance of $-\mathrm{OH}$ functional groups that can form hydrogen bonds with water and with each other, ${ }^{4,5}$ and that can subsequently form a tightly bound hydration layer to resist biomolecule adsorption. However, a number of studies have reported ${ }^{6,7}$ poor antifouling performance of polyHEMA-based materials in undiluted human blood serum and plasma. When these hydrophilic materials are in contact with complex media, none of these materials can retain their nonfouling properties, most likely because of the complex physicochemical interactions between proteins and surfaces.

\section{SECOND GENERATION: PEGYLATED SYSTEMS}

Poly(ethylene glycol) (PEG)- or oligo(ethylene glycol) (OEG)modified surfaces have been extensively studied for their nonfouling properties to resist protein adsorption and cell adhesion. The steric exclusion effect has been considered as one of the reasons for the resistance of PEG polymers (for example, poly(ethylene glycol) methacrylate (PEGMA)) to protein adsorption. ${ }^{8,9}$ Recent studies of OEG self-assembled monolayers showed that an appropriate surface density of OEG chains is needed for surface resistance to protein adsorption, and a tightly bound water layer around the OEG chains is 
Anti-Fouling Polymers

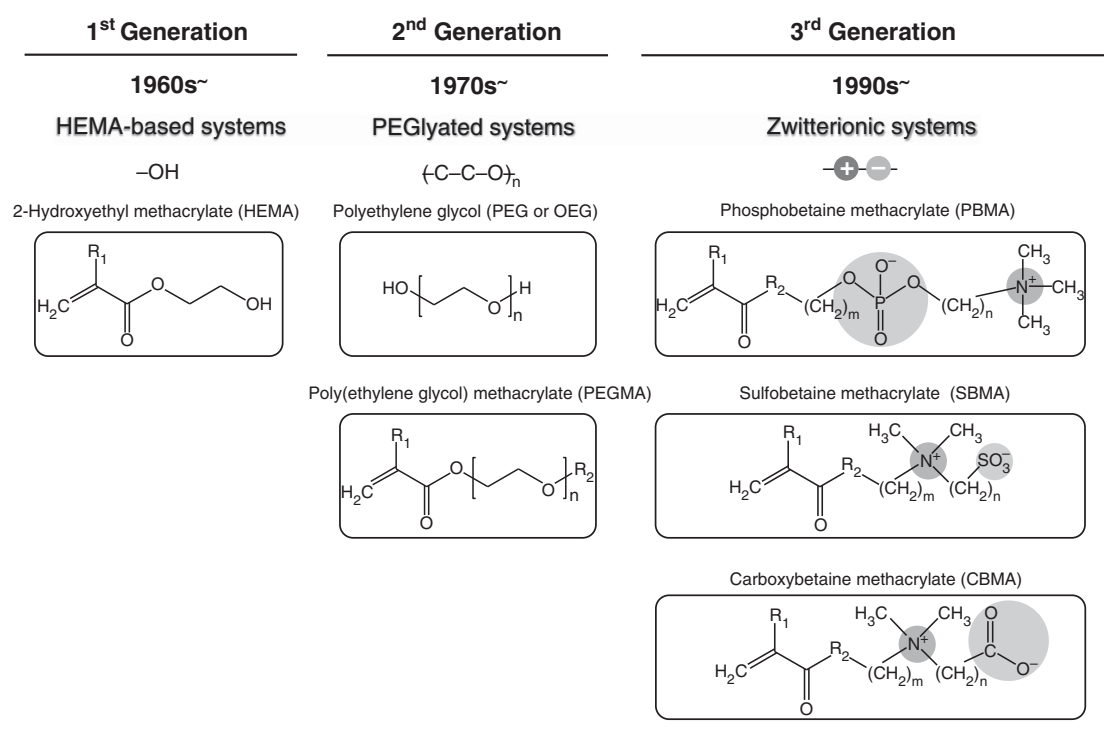

Figure 1 Evolution of antifouling materials. A full color version of this figure is available at Polymer Journal online.

primarily responsible for large repulsive hydration forces. ${ }^{10}$ However, because PEG and OEG groups decompose in the presence of oxygen and transition metal ions found in most biochemically relevant solutions, PEGylated surfaces face the problem of chemical stability. ${ }^{11-13}$ It has also been shown that grafted PEG brushes lose their protein-repulsive properties at physiological temperature. ${ }^{14}$ As a result, significant effort has been invested in the search for alternative antifouling materials with improved stability compared with PEG/OEG, and this work has resulted in substantial progress toward developing improved materials.

\section{THIRD GENERATION: ZWITTERIONIC SYSTEMS}

\section{Phosphobetaine}

Scientists began to develop zwitterionic antifouling materials based on the natural bio-inert theory that was inspired by the cell membrane. Zwaal et al. ${ }^{15}$ reported in 1997 that the inner membrane of red blood cells caused a thrombogenic response, whereas the outer layer did not. The proposed explanation was that the lipid components constituting the outside surface of the red blood cell membrane are mainly zwitterionic phospholipids (such as phosphorylcholine (PC)), whereas the inner components are negatively charged (such as phosphatidylserine). Inspired by the natural cell membrane lipid research, numerous biomimetic materials were subsequently developed. In 2003, the research group of Professor Lloyd ${ }^{16}$ designed PC-based polymers that have been used in a variety of medical device applications to improve biocompatibility.

Kadoma and colleagues ${ }^{17}$ and Ishihara et al. ${ }^{18}$ developed a new system. First, Kadoma and colleagues ${ }^{17}$ explored new synthetic routes for polymerizing MPC. The MPC structure is composed of a methacrylate and PC head group, and the side chain consists of a phosphate anion and a quaternary ammonium cation. Unlike the nonfouling hydrophilic-based materials, this zwitterionic structure could induce electrostatic interactions with the surrounding water molecules. However, the synthesis of MPCs was rather difficult and complex, and sufficient amounts of pure MPC could not be obtained. Later, Ishihara et al. ${ }^{18}$ established a refined and complete process for the synthesis and purification of MPC. This achievement led to considerable progress in the development of MPC polymers as biomaterials. MPC polymers are soluble in water, and their functionality can be easily adjusted by changing the structure and fraction of the comonomers. ${ }^{19-21}$

\section{Sulfobetaine}

The work on MPC-based surfaces was the first step toward the development of zwitterionic antifouling materials and introduced the importance of dual ions. However, MPC has some disadvantages, such as a rather complex synthetic route and low yields, both of which result in a high selling price. Therefore, other types of zwitterionic polymers have been explored, and those based on sulfobetaines have been particularly important. The sulfobetaine monomer with a quaternary ammonium cation and sulfonate anion on the same side chain closely resembles 2 -aminoethanesulfonic acid but is typically easier to handle than phosphorylcholine. Thus, the resulting polymers are generally easier to synthesize than the corresponding phosphorylcholine analogs. Recently, polySBMA, which has a methacrylate main chain and an analog of the taurine betaine as the pendant group $\left(\mathrm{CH}_{2} \mathrm{CH}_{2} \mathrm{~N}^{+}\left(\mathrm{CH}_{3}\right)_{2}-\mathrm{CH}_{2} \mathrm{CH}_{2} \mathrm{CH}_{2} \mathrm{SO}_{3}^{-}\right)$, has become the most widely studied zwitterionic polymer because of its ease of production and applicability. ${ }^{2,22}$ In addition, polysulfobetaines are not only biomimetic but are also biocompatible and noncytotoxic, as their endotoxin levels were found to be acceptable for in vivo implantation according to Zhang et al. ${ }^{23}$ The bio-inert nature of zwitterionic polySBMA polymers has been attributed to their strong hydration capabilities and the tightly bound, structured water layer around the zwitterionic pendant groups resulting from electrostatically induced hydration that plays an important role in achieving interfacial bioadhesion resistance. ${ }^{24,25}$

\section{Carboxybetaine}

Another zwitterionic material is carboxybetaine methacrylate that has side chains composed of cationic quaternary ammonium and anionic carboxylate functional groups. This material emerged in the antifouling field in 2003. The difference between carboxybetaine and sulfobetaine is that its negatively charged groups are based on the carboxylic acid $(-\mathrm{COOH})$ moiety. The structure of carboxybetaine is similar to that of glycine betaine that is one of the compatible solutes 
and is essential to the osmotic regulation of living organisms. ${ }^{26}$ In recent years, Jiang and colleagues ${ }^{27,28}$ found that zwitterionic carboxybetaine methacrylate is also a very good zwitterionic antifouling biomaterial. Carboxybetaine methacrylate may have wider biorecognition applications owing to the carboxylic acid groups that can be easily converted into other functional groups. The dual-functional poly(carboxybetaine) combines both ultralow fouling and functionalization with abundant bio-recognition elements $^{28-30}$ immobilized in one material, and this has not been achieved by any other material. Another unique aspect of poly(carboxybetaine) is that it can be prepared in a hydrolyzable form by replacing the carboxylate anion with an ester group. ${ }^{31}$ The esters can be converted into nontoxic and nonfouling zwitterionic polymers upon their hydrolysis.

\section{MOLECULAR DESIGN OF ZWITTERIONIC POLYSBMA-GRAFTED INTERFACES}

Apart from the design of functional materials, the molecular design of polymer brushes also plays an important role in achieving nonfouling properties on a surface. Thus, if polySBMA-grafted surfaces are well controlled, they should be able to achieve superb fouling resistance. $22,27,32,33$ Figure 2 reveals the optimum design of a zwitterionic interface that exhibit excellent hemocompatibility at a molecular level.

\section{PACKING DENSITY}

The effect of different polySBMA packing densities on protein adsorption behavior was delineated by Chang et al. ${ }^{22}$ In this study, it was found that protein adsorption solely depends on polySBMA surface density. Lower protein adsorption was observed when SBMA surface coverage consisted of low-molecular-weight $\left(M_{w}\right)$ polymer chains. An increase in surface packing defects was observed when the interface was grafted with large $M_{w}$ copolymers that resulted in higher protein adsorption. This effect was because larger SBMA segments create cavities among themselves and cannot fully cover the surface, leading to protein adsorption. However, very low protein adsorption was achieved when the surface covered with larger $M_{w}$ copolymers was back-filled with a smaller $M_{w}$ copolymer. Thus, surface packing density plays a significant role in surface resistance to protein adsorption. A similar phenomenon was reported by Nagasaki and colleagues ${ }^{34}$ who showed that adding shorter PEG chains onto a surface grafted with longer PEG chains further reduced protein adsorption.

\section{GRAFTING COVERAGE}

Previous studies have shown that controlling the surface grafting coverage of zwitterionic polySBMA layers is important for a bloodcompatible interface. ${ }^{35,36}$ Chang et al. ${ }^{37}$ described that an increase in the thickness of the polySBMA brush (increased chain length) resulted in an increase in the surface hydration, as full surface coverage and uniformity of polySBMA brushes allow for complete water surface contact. ${ }^{37}$ It was found that the existence of sufficient hydration layers between grafted brushes allows for a well-extended configuration of individual polySBMA chains. This was the first report to show that surface-grafted polySBMA brushes with high grafting coverage can be used to attain excellent blood compatibility.

\section{POLYMER CHAIN CONFORMATION}

It is hypothesized that the nonfouling properties of polyzwitterionic materials are tightly correlated with the existence of a hydration layer near the surface because a tightly bound water layer forms a physical and energetic barrier to prevent protein adsorption on the surface. Although most water-soluble polymers can reduce protein adsorption to some extent, the best nonfouling ability can only be achieved when surface hydration and chain flexibility (that is, steric repulsion) work together. Recently, Sin et al. ${ }^{38}$ reported that the conformational structure of the anchoring agent assembly layer is a determining factor in the formation of flexible, well-spaced polySBMA brushes with greater hydration capacity and grafting coverage. ${ }^{38}$ For example, polySBMA brushes grafted from a dopamine-anchored surface are likely loosely packed on the molecular level, and this packing could lead to a thick hydration layer at the interface because there is more space for free water molecules to flow between the polymer brushes. The augmented interfacial hydration capacity and stronger repulsive hydration forces could thus result in an improved resistance to nonspecific bioadhesion.

\section{POLYMER CHAIN CHEMISTRY}

It has been suggested that neutral surfaces composed of positively and negatively charged pairs possess protein-resistant properties. ${ }^{39}$ Novel studies on the effects of the molecular arrangement of mixed charged groups on fouling-resistant performance were conducted in our

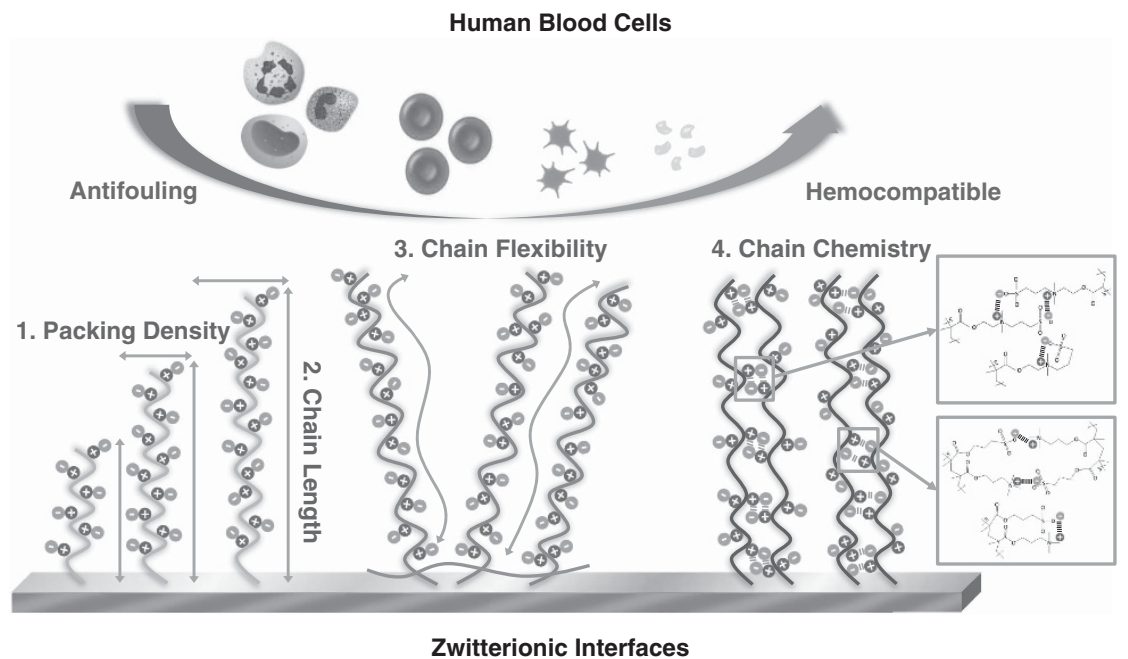

Figure 2 Molecular design of zwitterionic interfaces for excellent hemocompatibility. A full color version of this figure is available at Polymer Journal online. 
laboratory in 2010 and 2013. ${ }^{37,40}$ In these studies, it was demonstrated that copolymer brushes of poly([2-(methacryloyloxy) ethyl] trimethylammonium chloride-co-3-sulfopropyl methacrylate potassium salt) (poly(TMA-co-SA)) with a homogeneous charge balance, prepared from two oppositely charged compounds, are highly resistant to nonspecific protein adsorption. This resistance could be attributed to the distance between the two oppositely charged groups that are associated with the hydration-promoting effects on inter- and intramolecular interactions between copolymer brushes. These studies demonstrated that the spacing between two oppositely charged groups at the nanometer scale might be similar to that of the ethylene spacer between the two charged groups in the polySBMA side chains, resulting in the nonassociations of poly(TMA-co-SA) brushes.

\section{'INTELLIGENT' INTERFACES}

An 'intelligent interface' consisting of a thermoresponsive poly ( $N$-isopropylacrylamide)-block-poly(sulfobetaine methacrylate) (PNIPAAm- $b$-PSBMA) hydrogel that could respond to environmental stimuli over a small scale by changing its physical conformation (Figure 3) was investigated in our laboratory in 2009, 2010 and 2012. Our work reported on the tunable effects of PNIPAAm- $b$-PSBMA copolymer conformations on the correlations between chain associations and blood compatibility ${ }^{41}$ that exhibited controllable thermosensitive phase transition of lower critical solution temperature (LCST) and upper critical solution temperature (UCST) behaviors in an aqueous solution. ${ }^{42,43}$ It was reported that good blood compatibility was observed with zwitterionic polySBMA polymers above their UCSTs and nonionic polyNIPAAm polymers below their LCSTs in human blood.

As shown in Figure 3, the prepared copolymer PNIPAAm- $b$ PSBMA exhibited a doubly thermoresponsive transition behavior that resulted from the switchable nonionic/zwitterionic block selfassembly driven by the formation of inter- and intramolecular electrostatic interactions between SBMA segments of the zwitterionic sulfobetaine groups and the intramolecular hydrophobic interactions between NIPAAm segments of the nonionic isopropyl groups. At temperatures above the LCST, polyNIPAAm chains become hydrophobic, and their hydrogen bonding with water molecules weakens, resulting in the collapsing of the polyNIPAAm block and the precipitation of the polymer. Intramolecular hydrophobic interactions between the nonionic isopropyl moieties of polyNIPAAm drive the precipitation observed above the LCST. At temperatures below the UCST, polySBMA exists as a collapsed coil and precipitates in aqueous solution. This phenomenon, which is attributed to the strong mutual intra- and intermolecular associations of the zwitterionic groups induced by electrostatic interaction, gives rise to the loss of the nonfouling nature of polySBMA.

\section{CHRONOLOGICAL DEVELOPMENT OF ZWITTERIONIC INTERFACES AND MEMBRANES}

Protein adsorption on material surfaces causes serious biological reactions, such as thrombus formation, an immune response, complement activation, capsulation and so on. ${ }^{44,45}$ Thus, excellent nonspecific plasma protein-fouling resistance is one of the most important requirements for developing blood-contacting materials. In 2006, Professor Chang, Jiang and colleagues ${ }^{22}$ prepared well-defined diblock copolymers of polySBMA and poly(propylene oxide). These copolymers were physically immobilized onto a surface plasmon resonance sensor surface covered by methyl-terminated self-assembled monolayers, and protein adhesion on the adsorbed copolymers was evaluated in situ. The results revealed that the diblock copolymers possessing polySBMA can be eminently protein resistant when the surface SBMA density is well controlled. As thrombus formation is one of the most serious host responses to implanted materials, the platelet adhesion test was adopted as a recognized technique for estimating the blood compatibility of a prepared material surface. Our laboratory employed this test to evaluate the blood compatibility of a polySBMA-grafted surface. In our first study on antithrombogenic zwitterionization in 2008, SBMA was grafted from a bromide-covered gold surface through surface-initiated atom transfer radical polymerization to produce well-packed polymer brushes. ${ }^{46}$ From the results of both protein and platelet adsorption in vitro, it was demonstrated that a well-packed polySBMA-grafted surface exhibited stable and excellent blood compatibility at human body temperature.

Interestingly, zwitterionic polySBMA can present diverse forms, including soluble unimers or micelles in aqueous medium, adsorbed or grafted onto aqueous-solid interfaces, or crosslinked in the form of physical or chemical hydrogels. However, little is known about how polySBMA conformation affects the correlation between solution properties and blood compatibility. Accordingly, a systematic study on the molecular-weight dependence of hemocompatibility correlated with the solution properties of zwitterionic polySBMA polymers was reported in $2010 .{ }^{41}$ It was found that polySBMA polymers of increasing molecular weights had enhanced mutual intra- and interchain associations of the sulfobetaine groups in aqueous solution that increased their UCSTs associated with the nonfouling nature of the polySBMA suspension. Studies have also reported that physical micellization occurs in synthesized diblock copolymers with thermoresponsive and zwitterionic properties. ${ }^{47,48}$ In 2009 and 2010,

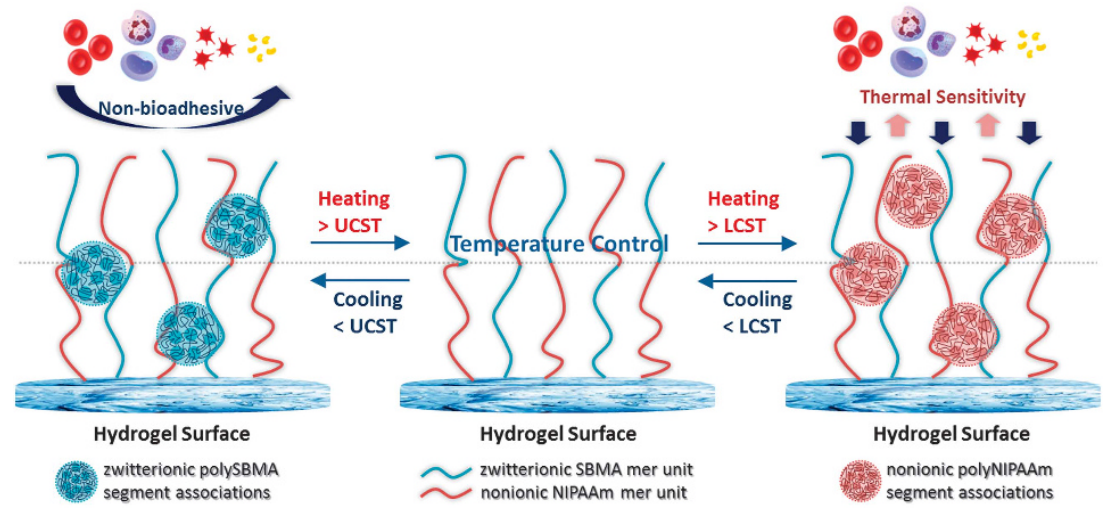

Figure 3 Responses of 'intelligent' interfaces in conjunction with thermal stimuli. 
a new class of 'smart' copolymers consisting of PNIPAAm- $b$-PSBMA, which were prepared from the combination of zwitterionic polySBMA and nonionic thermoresponsive polyNIPAAm, was reported. These materials exhibited controllable thermosensitive phase transition of LCST and UCST behaviors in an aqueous solution. ${ }^{42,43}$ These studies proposed that a zwitterionic copolymer containing nonionic polyNIPAAm is a potential thermoresponsive biomaterial that can provide a coated surface with tunable, stimulus-responsive properties for use in a physiologic environment. In addition, the unique characteristics of thermoresponsive polyNIPAAm hydrogels incorporating zwitterionic polySBMA can be exploited to regulate cell attachment or detachment on the surfaces using temperatures above or below the LCST of the copolymeric gels. In 2012, our laboratory investigated diblock copolymers containing polyNIPAAm and polySBMA as a new class of intelligent, 'schizophrenic' polymers, exhibiting good hemocompatibility over a wide range of temperatures from normal blood storage to the human physiologic environment. ${ }^{49}$ This reversible morphology variation was attributed to the reorganization of hydrophobic/hydrophilic balance in response to the $\mathrm{pH}$ and temperature, thus maximizing the nonfouling property.

Recently, it has been reported that nonfouling surfaces with homogeneous charge balances, prepared by mixing two oppositely charged compounds, are highly resistant to nonspecific single-protein adsorption as well. ${ }^{39,50}$ However, these studies did not extend to the use or evaluation of these mixed-charge surfaces as human bloodcontacting materials. In our pseudo-zwitterionization study in 2010, ${ }^{24}$ mixed-charge copolymer brushes of poly(TMA-co-SA), which are similar to zwitterionic homopolymer brushes, were synthesized for similar applications requiring protein-fouling resistance. However, under all conditions tested, it was found that the nonfouling surfaces prepared from the zwitterionic structure of polySBMA brushes were better than the mixed-charge structure of poly(TMA-co-SA) copolymer brushes. The general concept of preparing new nonfouling materials from common oppositely charged groups is to carry out the synthesis in an easy way, but the separation between the two charged sites must be considered along with overall charge neutrality of copolymer brushes.

In 2012, our laboratory reported for the first time that surfacegrafted polymer brushes of polySBMA with high grafting coverage and overall charge neutrality can be used to provide the best preservation capacity for hematopoietic stem and progenitor cells stored in umbilical cord blood solution. ${ }^{37}$ Their superior capacity for hematopoietic stem and progenitor cell preservation could be because of the high surface roughness and flexible chain mobility of hydrated polySBMA brushes. It is also desirable to develop a copolymer suitable for nonfouling coatings on charged surfaces as many biomaterials surfaces are polar/hydrophilic but not blood-inert. ${ }^{51,52}$ Hence, a strategy to create blood-inert surfaces in human whole blood via ion-pair anchoring of zwitterionic copolymer brushes and a systematic study of how well-defined chain lengths and wellcontrolled surface packing densities affect blood compatibility were reported in 2013.40 In this study, ionic-zwitterionic block copolymers poly(3-sulfopropyl methacrylate potassium salt)-blockpoly(sulfobetaine methacrylate) (PSA- $b$-PSBMA) were anchored onto charged surfaces through a robust charge-controlled grafting approach. This ion-pair anchoring design facilitates a convenient, stable and efficient immobilization of ionic-zwitterionic copolymers directly onto charged surfaces via charge pairing.

A study of surface-zwitterionized stainless steel implant materials, which were prepared via catechol dopamine- and organosilanesurfaced immobilization of zwitterionic polysulfobetaine brushes, for general bioadhesive control was reported in 2014. ${ }^{38}$ Excellent anti-bioadhesive performance of stainless steel surfaces grafted with flexible-type zwitterionic polymer brushes via catechol dopaminesurfaced immobilization was achieved with an almost undetectable level of protein adsorption, no blood-cell adhesion and activation, no tissue-cell attachment and no bacterial adhesion. This excellent bioadhesion resistance could be attributed to the anchoring agent, the polydopamine assembly layer, that acts as a vital component for forming flexible, well-spaced polySBMA brushes with greater hydration capacity and grafting coverage.

Apart from zwitterionic interfaces, our research group has also studied the surface zwitterionization of several polymeric membranes. In the early work on developing zwitterionic membranes, interpenetrating polymer networks were prepared by modifying segmented polyurethane (SPU) films with a crosslinked zwitterionic SBMA polymer. ${ }^{53}$ It was found that the uniform distribution of SBMA units within SPU films can effectively resist nonspecific protein adsorption and retain the high mechanical strength that is inherent to the base SPU films. In 2009 and 2011, a new membrane zwitterionization process based on grafting a zwitterionic polySBMA layer onto the poly(vinylidene fluoride) (PVDF) membrane surface using an integrated plasma technique was reported. ${ }^{25,54}$ The results indicated that PVDF- $g$-polySBMA membranes presented a fouling resistance for plasma proteins and blood platelets that greatly relied on the surface hydrophilicity and

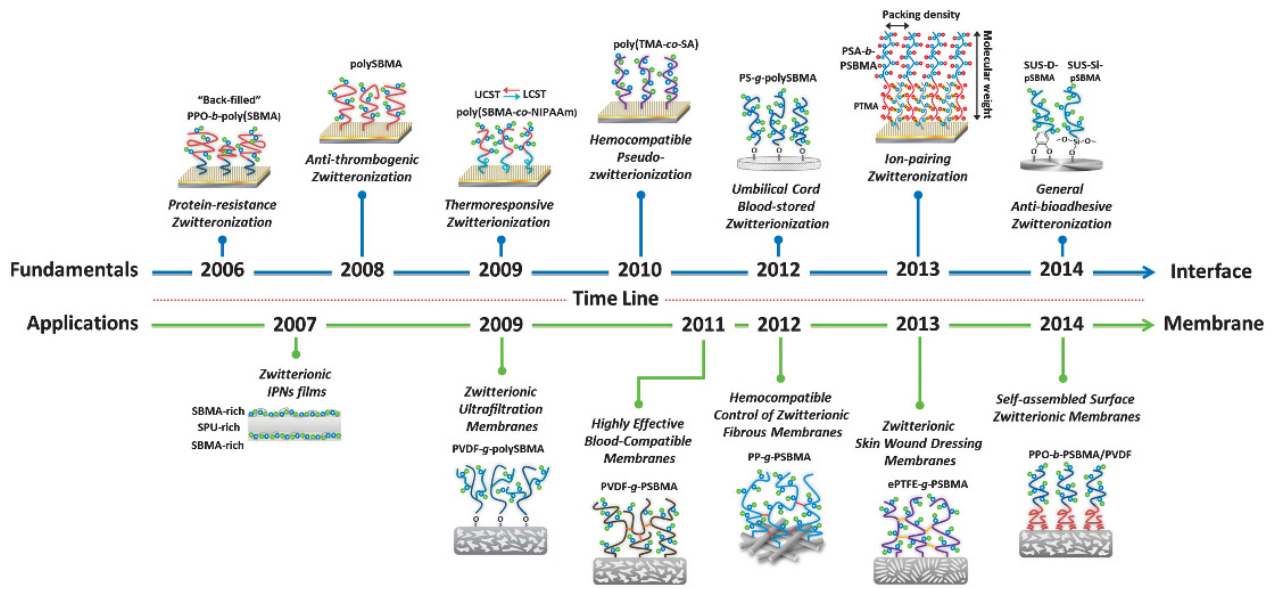

Figure 4 Chronology of significant developments of zwitterionic interfaces and membranes in our research group. 


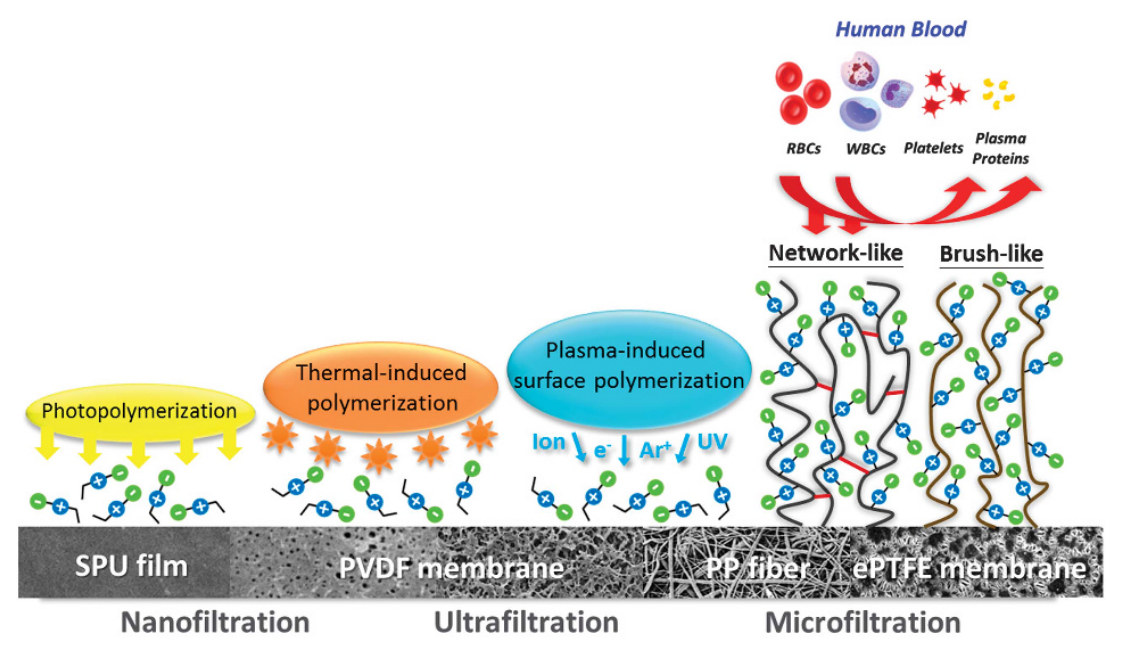

Zwitterionic Membranes

Figure 5 Correlation between polymeric membrane surface modification and the conformation of grafted poly(sulfobetaine methacrylate) (polySBMA) chains.

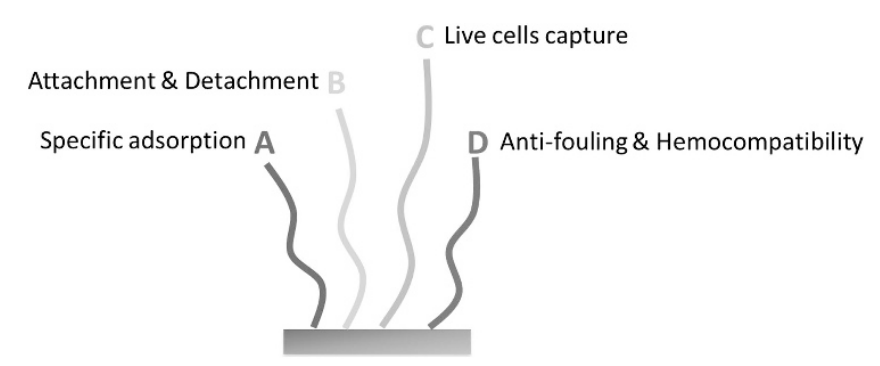

Figure 6 Multifunctional interfaces/membranes for specific applications. A full color version of this figure is available at Polymer Journal online.

charge bias of the zwitterionic polySBMA layer. In the following years, zwitterionic polySBMA was grafted onto polypropylene fibrous membranes $^{55}$ and expanded poly(tetrafluoroethylene) (ePTFE) membranes ${ }^{56}$ via atmospheric plasma-induced graft polymerization. The polySBMA-grafted ePTFE membranes were investigated as a wound healing dressing, and they provided a moist environment, prevented biofouling and allowed deep skin wounds to completely heal after 2 weeks.

Nevertheless, plasma surface modification is not practical for the large-scale production of zwitterionic membranes. Consequently, in 2014, Antoine et al. employed a fast and convenient coating approach based on hydrophobic-driven self-assembly to coat PVDF microfiltration membranes with the diblock copolymer poly(propylene oxide)-block-poly(sulfobetaine methacrylate) (PPO- $b$-PSBMA). ${ }^{57}$ Excellent blood compatibility was achieved using this method by adjusting the anchoring structures of the zwitterionic copolymer layers. The chronology of significant developments of zwitterionic interfaces and membranes in our laboratory is summarized in Figure 4.

\section{DEVELOPMENT OF NONIONIC AND ZWITTERIONIC-GRAFTED MEMBRANE}

The fundamental research on zwitterionic polymers applied to interfaces provided the concept of zwitterionization. It not only successfully solved the problem of serious biofouling on biomaterial interfaces but also significantly improved surface hemocompatibility. However, it should be noted that besides biomaterial interfaces, polymeric membranes also play an important role in developing medical devices used in hemodialysis, blood filtration and the heart-lung machine. In the following section, correlations between the polymeric membrane surface modification approach and the conformation of grafted polySBMA chains are discussed. These correlations are elaborated in Figure 5.

Our first zwitterionic polymeric membrane was developed in 2007 via a simple photopolymerization process. ${ }^{53}$ In this work, SPU films were modified with a crosslinked SBMA to form interpenetrating polymer networks that could effectively suppress nonspecific protein adsorption when the distribution of SBMA units within the SPU film was well controlled. In 2008, nonionic PEGMA was successfully grafted from microporous $\mathrm{PVDF}^{58}$ and expanded ePTFE ${ }^{59}$ membranes via thermal-induced graft polymerization using $\mathrm{O}_{3} / \mathrm{O}_{2}$ or $\mathrm{H}_{2}$ plasma pretreatment. The results indicated that PEGMA-grafted membranes significantly reduced irreversible membrane fouling. Thereafter, similar $\mathrm{O}_{3} / \mathrm{O}_{2}$ pretreatment and surface-initiated atom transfer radical polymerization approaches were adopted in 2009 to prepare a zwitterionic polySBMA-grafted PVDF ultrafiltration membrane ${ }^{54}$ that exhibited an extremely low biofouling filtration performance.

Nevertheless, the above preparation processes were somewhat complicated. Thus, in 2009, a new surface modification technique termed low-pressure plasma-induced graft polymerization was developed to graft PEGMA onto PVDF membranes, and the results showed better antifouling efficiency compared with PEGMA-grafted PVDF membranes prepared by thermal-induced polymerization and surface-initiated atom transfer radical polymerization. ${ }^{60}$ The effect of low-pressure and atmospheric plasma graft polymerization on the conformation structure of grafted-PEGMA was further investigated in $2011 .^{61}$ The results demonstrated that the brush-like PEGMA structure produced by atmospheric plasma polymerization had a higher hydration capacity and exhibited better fouling suppression than the network-like PEGMA structure prepared by the lowpressure plasma approach. Therefore, in the following years, zwitterionic polySBMA were grafted onto PVDF ultrafiltration membranes, ${ }^{25}$ polypropylen fibrous membranes ${ }^{55}$ and expanded PTFE membranes ${ }^{56}$ using atmospheric plasma-induced graft polymerization. Collectively, these studies suggest that zwitterionic membranes generated by controlling the charge neutrality of grafted brush-like polySBMA structures have great potential for the surface 
modification of general hydrophobic membranes used in human blood-contacting devices.

\section{CONCLUSIONS AND PERSPECTIVES}

Zwitterionic polysulfobetaines are very promising as next-generation hemocompatible biomaterials because of their excellent nonfouling properties in complex media. As revealed in Figure 6, future directions are aimed toward the development of multifunctional zwitterionic interfaces/membranes for specific applications, such as specific biomolecule adsorption, cell attachment and detachment and live cell capture, all while maintaining their excellent antibiofouling properties.

\section{ACKNOWLEDGEMENTS}

We express our sincere gratitude to the Center-of-Excellence (COE) Program on Membrane Technology from the Ministry of Education (MOE), ROC, the project of Outstanding Professor Research Program in the Chung Yuan Christian University (CYCU-00RD-RA002-11757) and the National Science Council (NSC 100-2628-E-033-001-MY3 and NSC 102-2221-E-033-009-MY3) for their financial support.

1 Chen, S., Cao, Z. \& Jiang, S. Ultra-low fouling peptide surfaces derived from natura amino acids. Biomaterials 30, 5892-5896 (2009).

2 Zhang, Z., Chao, T., Chen, S. \& Jiang, S. Superlow fouling sulfobetaine and carboxybetaine polymers on glass slides. Langmuir 22, 10072-10077 (2006).

3 Ostuni, E., Chapman, R. G., Holmlin, R. E., Takayama, S. \& Whitesides, G. M. A survey of structure-property relationships of surfaces that resist the adsorption of protein. Langmuir 17, 5605-5620 (2011).

4 Leduc, E. H. \& Holt, S. J. Hydroxypropyl methacrylate: a new water-miscible embedding medium for electron microscopy. J. Cell Biol. 26, 137 (1965).

5 Lewis, A. L., Cumming, Z. L., Goreish, H. H., Kirkwood, L. C., Tolhurst, L. A. \& Stratford, P. W. Crosslinkable coatings from phosphorylcholine-based polymers. Biomaterials 22, 99-111 (2001).

6 Mrabet, B., Nguyen, M. N., Majbri, A., Mahouche, S., Turmine, M., Bakhrouf, A. \& Chehimi, M. M. Anti-fouling poly(2-hydoxyethyl methacrylate) surface coatings with specific bacteria recognition capabilities. Surf. Sci. 603, 2422-2429 (2009).

7 Yoshikawa, C., Goto, A., Tsujii, Y., Fukuda, T., Kimura, T., Yamamoto, K. \& Kishida, A. Protein repellency of well-defined, concentrated poly(2-hydroxyethyl methacrylate) brushes by the size-exclusion effect. Macromolecules 39, 2284-2290 (2006).

$8 \mathrm{Ma}, \mathrm{H}$., Hyun, J., Stiller, P. \& Chilkoti, A. "Non-fouling" oligo(ethylene glycol)functionalized polymer brushes synthesized by surface-initiated atom transfer radical polymerization. Adv. Mater. 16, 338-341 (2004).

9 Zheng, J., Li, L., Tsao, H. K., Sheng, Y. J., Chen, S. \& Jiang, S. Strong repulsive forces between protein and oligo (ethylene glycol) self-assembled monolayers: a molecular simulation study. Biophys. J. 89, 158-166 (2005).

10 Zheng, J., Li, L., Chen, S. \& Jiang, S. Molecular simulation study of water interactions with oligo (ethylene glycol)-terminated alkanethiol self-assembled monolayers. Langmuir 20, 8931-8938 (2004).

11 Ostuni, E., Chapman, R. G., Holmlin, R. E., Takayama, S. \& Whitesides, G. M. A survey of structure-property relationships of surfaces that resist the adsorption of protein. Langmuir 17, 5605-5620 (2001).

12 Shen, M. C., Martinson, L., Wagner, M. S., Castner, D. G., Ratner, B. D. \& Horbett, T. A. PEO-like plasma polymerized tetraglyme surface interactions with leukocytes and proteins: in vitro and in vivo studies. J. Biomater. Sci., Polym. Ed. 13, 367-390 (2002)

13 Luk, Y. Y., Kato, M. \& Mrksich, M. Self-assembled monolayers of alkanethiolates presenting mannitol groups are inert to protein adsorption and cell attachment. Langmuir 16, 9604-9608 (2000).

14 Leckband, D., Sheth, S. \& Halperin, A. Grafted poly(ethylene oxide) brushes as nonfouling surface coatings. J. Biomater Sci. Polym. Ed. 10, 1125-1147 (1999).

15 Zwaal, R. F. A. \& Schroit, A. J. Pathophysiologic implications of membrane phospholipid asymmetry in blood cells. Blood 89, 1121-1132 (1997).

16 Long, S. F., Clarke, S., Davies, M. C., Lewis, A. L., Hanlon, G. W. \& Lloyd, A. W. Controlled biological response on blends of a phosphorylcholine-based copolymer with poly(butyl methacrylate). Biomaterials 24, 4115-4121 (2003).

17 Kadoma, Y., Nakabayashi, N., Masuhara, E. \& Yamauchi, J. Synthesis and hemolysis test of the polymer containing phosphorylcholine groups. Kobunshi Ronbunshu 35 , 423-427 (1978).

18 Ishihara, K., Ueda, T. \& Nakabayashi, N. Preparation of phospholipid polylners and their properties as polymer hydrogel membranes. Polym. J. 22, 355-360 (1990).

19 Ishihara, K., Tsuji, T., Kurosaki, K. \& Nakabayashi, N. Hemocompatibility on graft copolymers composed of poly(2-methacryloyloxyethyl phosphorylcholine) side chain and poly(n-butyl methacrylate) backbone. J. Biomed. Mater. Res. 28, 225-232 (1994).

20 Watanabe, J., Eriguchi, T. \& Ishihara, K. Cell adhesion and morphology in porous scaffold based on enantiomeric poly(lactic acid) graft-type phospholipid polymers. Biomacromolecules 3, 1375-1383 (2002).

21 Iwasaki, Y. \& Ishihara, K. Cell membrane-inspired phospholipid polymers for developing medical devices with excellent biointerfaces. Sci. Technol. Adv. Mater. 13, 64-101 (2012)

22 Chang, Y., Chen, S., Zhang, Z. \& Jiang, S. Y. Highly protein-resistant coatings from well-defined diblock copolymers containing sulfobetaines. Langmuir 22, 2222-2226 (2006)

23 Zhang, Z., Chao, T., Liu, L., Cheng, G., Ratner, B. D. \& Jiang, S. Zwitterionic hydrogels: an in vivo implantation study. J. Biomater. Sci. Polym. Ed. 20, 1845-1859 (2009).

24 Chang, Y., Shu, S. H., Chu, C. W., Shih, Y. J., Ruaan, R. C. \& Chen, W. Y. Hemocompatible mixed-charge copolymer brushes of pseudo-zwitterionic surfaces resistant to nonspecific plasma protein fouling. Langmuir 26, 3522-3530 (2010)

25 Chang, Y., Chang, W. J., Shih, Y. J., Wei, T. C. \& Hsiue, G. H. Zwitterionic sulfobetaine grafted poly(vinylidene fluoride) membrane with highly effective blood compatibility via atmospheric plasma-induced surface copolymerization. ACS Appl. Mater. Interfaces 3, 1228-1237 (2011)

26 Nyyssola, A. Pathways of glycine betaine synthesis in two extremely halophilic bacteria Actinopolyspora Halophila and Ectothiorhodospira Halochloris. PhD thesis, Helsinki University of Technology, Finland (2001).

27 Ladd, J., Zhang, Z., Chen, S., Hower, J. C. \& Jiang, S. Zwitterionic polymers exhibiting high resistance to nonspecific protein adsorption from human serum and plasma. Biomacromolecules 9, 1357-1361 (2008).

28 Vaisocherova, H., Yang, W., Zhang, Z., Cao, Z., Cheng, G., Piliarik, M., Homola, J. \& Jiang, S. Ultralow fouling and functionalizable surface chemistry based on a zwitterionic polymer enabling sensitive and specific protein detection in undiluted blood plasma. Anal. Chem. 80, 7894-7901 (2008).

29 Zhang, Z., Chen, S. \& Jiang, S. Dual-functional biomimetic materials: nonfouling poly(carboxybetaine) with active functional groups for protein immobilization. Biomacromolecules 7, 3311-3315 (2006).

30 Yang, W., Xue, H. \& Jiang, S. Pursuing "zero" protein adsorption of poly (carboxybetaine) from undiluted blood serum and plasma. Langmuir 25, 11911-11916 (2009).

31 Jiang, S. \& Cao, Z. Ultralow-fouling, functionalizable, and hydrolysable zwitterionic materials and their derivatives for biological applications. Adv. Mater. 22, 920-932 (2010).

32 Feng, W., Brash, J. L. \& Zhu, S. Non-biofouling materials prepared by atom transfer radical polymerization grafting of 2-methacryloloxyethyl phosphorylcholine: separate effects of graft density and chain length on protein repulsion. Biomaterials 27, 847-855 (2006)

33 Yang, W., Chen, S., Cheng, G., Vaisocherova, H., Xue, H., Li, W., Zhang, J. \& Jiang, S Film thickness dependence of protein adsorption from blood serum and plasma onto poly(sulfobetaine)-grafted surfaces. Langmuir 24, 9211-9214 (2008).

34 Uchida, K., Otsuka, H., Kaneko, M., Kataoka, K. \& Nagasaki, Y. A reactive poly(ethylene glycol) layer to achieve specific surface plasmon resonance sensing with a high S/N ratio: the substantial role of a short underbrushed PEG layer in minimizing nonspecific adsorption. Anal. Chem. 77, 1075-1080 (2005).

35 Zhang, Z., Zhang, M., Chen, S., Horbett, T. A., Ratner, B. D. \& Jiang, S. Blood compatibility of surfaces with superlow protein adsorption. Biomaterials 28 4285-4291 (2008).

36 Zhang, Z., Chen, S. F., Chang, Y. \& Jiang, S. Y. Surface grafted sulfobetaine polymers via atom transfer radical polymerization as superlow fouling coatings. J. Phys. Chem. B 110, 10799-10804 (2006).

37 Chang, Y., Chang, Y., Higuchi, A., Shih, Y. J., Li, P. T., Chen, W. Y., Tsai, E. M. \& Hsiue, G. H. Bioadhesive control of plasma proteins and blood cells from umbilical cord blood onto the interface grafted with zwitterionic polymer brushes. Langmuir $\mathbf{2 8}$, 4309-4317 (2012)

38 Sin, M. C., Sun, Y. M. \& Chang, Y. Zwitterionic-based stainless steel with well-defined polysulfobetaine brushes for general bioadhesive control. ACS Appl. Mater. Interfaces 6, 861-873 (2014)

39 Holmlin, R. E., Chen, X. X., Chapman, R. G., Takayama, S. \& Whitesides, G. M. Zwitterionic SAMs that resist nonspecific adsorption of protein from aqueous buffer Langmuir 17, 2841-2850 (2001).

40 Chang, Y., Shih, Y. J., Lai, C. J., Kung, H. H. \& Jiang, S. Ion-pair anchoring of selfassembled zwitterionic copolymer surfaces with highly controlled blood compatibility. Adv. Funct. Mater. 23, 1100-1110 (2013).

41 Shih, Y. J. \& Chang, Y. Tunable blood compatibility of polysulfobetaine from controllable molecular-weight dependence of zwitterionic nonfouling nature in aqueous solution. Langmuir 26, 17286-17294 (2010)

42 Chang, Y., Chen, W. Y., Yandi, W., Shih, Y. J., Chu, W. L., Liu, Y. L., Ruaan, R. C. \& Higuchi, A. Dual-thermoresponsive phase behavior of blood compatible zwitterionic copolymers containing nonionic poly(N-isopropyl acrylamide). Biomacromolecules $\mathbf{1 0}$, 2092-2100 (2009)

43 Chang, Y., Yandi, W., Chen, W. Y., Shih, Y. J., Yang, C. C., Chang, Y., Ling, Q. D. \& Higuchi, A. Tunable bioadhesive copolymer hydrogels of thermoresponsive poly (N-isopropylacrylamide) containing zwitterionic polysulfobetaine. Biomacromolecules $11,1101-1110$ (2010).

44 Brash, J. L. \& Horbett, T. A. Proteins at Interfaces: Physicochemical and Biochemical Studies (American Chemical Society, Washington, DC, USA, 1987) 
45 Horbett, T. A. \& Brash, J. L. Proteins at Interfaces II: Fundamentals and Applications (American Chemical Society, Washington, DC, USA, 1995).

46 Chang, Y., Liao, S. C., Higuchi, A., Ruaan, R. C., Chu, C. W. \& Chen, W. Y. A highly stable nonbiofouling surface with well-packed grafted zwitterionic polysulfobetaine for plasma protein repulsion. Langmuir 24, 5453-5458 (2008).

47 Arotcarena, M., Heise, B., Ishaya, S. \& Laschewsky, A. Switching the inside and the outside of aggregates of water-soluble block copolymers with double thermoresponsivity. J. Am. Chem. Soc. 124, 3787-3793 (2002).

48 Maeda, Y., Mochiduki, H. \& Ikeda, I. Hydration changes during thermosensitive association of a block copolymer consisting of LCST and UCST blocks. Macromol. Rapid Commun. 25, 1330-1334 (2004).

49 Shih, Y. J., Chang, Y., Deratani, A. \& Damien, Q. "Schizophrenic" hemocompatible copolymers via switchable thermoresponsive transition of nonionic/zwitterionic block self-assembly in human blood. Biomacromolecules 13, 2849-2858 (2012).

50 Chen, S. F. \& Jiang, S. Y. An new avenue to nonfouling material. Adv. Mater. 20, 335-338 (2008).

51 Ratner, B. D., Hoffman, A. S., Schoen, F. J. \& Lemons, J. E. Biomaterials Science, an Introduction to Materials in Medicine (Elsevier, Amsterdam, Netherlands, 2004).

52 Kuo, W. H., Wang, M. J., Chien, H. W., Wei, T. C., Lee, C. \& Tsai, W. B. Surface modification with poly(sulfobetaine methacrylate-co-acrylic acid) to reduce fibrinogen adsorption, platelet adhesion, and plasma coagulation. Biomacromolecules 12, 4348-4356 (2011).

53 Chang, Y., Chen, S., Yu, Q., Zhang, Z., Bernards, M. \& Jiang, S. Y. Development of biocompatible interpenetrating polymer networks containing a sulfobetaine-based polymer and a segmented polyurethane for protein resistance. Biomacromolecules $\mathbf{8}$, 122-127 (2007).

54 Chiang, Y. C., Chang, Y., Higuchi, A., Chen, W. Y. \& Ruaan, R. C. Sulfobetaine-grafted poly(vinylidene fluoride) ultrafiltration membranes exhibit excellent antifouling property. J. Membr. Sci. 339, 151-159 (2009).
55 Chen, S. H., Chang, Y., Lee, K. R., Wei, T. C., Higuchi, A., Ho, F. M., Tsou, C. C., Ho, H. T. \& Lai, J. Y. Hemocompatible control of sulfobetaine-grafted polypropylene fibrous membranes in human whole blood via plasma-induced surface zwitterionization. Langmuir 28, 17733-17742 (2012).

56 Jhong, J. F., Venault, A., Hou, C. C., Chen, S. H., Wei, T. C., Zheng, J., Huang, J. \& Chang, Y. Surface zwitterionization of expanded poly(tetrafluoroethylene) membranes via atmospheric plasma-induced polymerization for enhanced skin wound healing. ACS Appl. Mater. Interfaces 5, 6732-6742 (2013).

57 Venault, A., Chang, Y., Yang, H. S., Lin, P. Y., Shih, Y. J. \& Higuchi, A. Surface self-assembled zwitterionization of poly(vinylidene fluoride) microfiltration membranes via hydrophobic-driven coating for improved blood compatibility. J. Membr. Sci. 454, 253-263 (2014).

58 Chang, Y., Shih, Y. J., Ruaan, R. C., Higuchi, A., Chen, W. Y. \& Lai, J. Y. Preparation of poly(vinylidene fluoride) microfiltration membrane with uniform surface-copolymerized poly(ethylene glycol) methacrylate and improvement of blood compatibility. J. Membr. Sci. 309, 165-174 (2008).

59 Chang, Y., Cheng, T. Y., Shih, Y. J., Lee, K. R. \& Lai, J. Y. Biofouling-resistance expanded poly(tetrafluoroethylene) membrane with a hydrogel-like layer of surfaceimmobilized poly(ethylene glycol) methacrylate for human plasma protein repulsions. J. Membr. Sci. 323, 77-84 (2008).

60 Chang, Y., Ko, C. Y., Shih, Y. J., Quémener, D., Deratani, A., Wei, T. C., Wang, D. M. \& Lai, J. Y. Surface grafting control of PEGylated poly(vinylidene fluoride) antifouling membrane via surface-initiated radical graft copolymerization. J. Membr. Sci. 345, 160-169 (2009).

61 Chang, Y., Shih, Y. J., Ko, C. Y., Jhong, J. F., Liu, Y. L. \& Wei, T. C. Hemocompatibility of poly(vinylidene fluoride) membrane grafted with network-like and brush-like antifouling layer controlled via plasma-induced surface PEGylation. Langmuir 27, 5445-5455 (2011). 